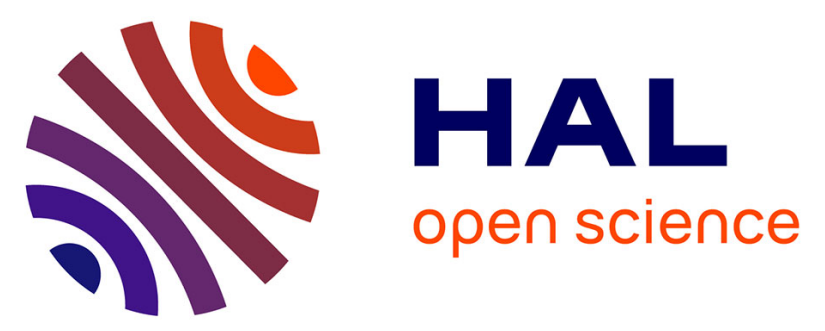

\title{
A Framework for Concurrent Design of Metamodels and Diagrams: Towards an Agile Method for the Synthesis of Domain Specific Graphical Modeling Languages
}

François Pfister, Marianne Huchard, Clémentine Nebut

\section{- To cite this version:}

François Pfister, Marianne Huchard, Clémentine Nebut. A Framework for Concurrent Design of Metamodels and Diagrams: Towards an Agile Method for the Synthesis of Domain Specific Graphical Modeling Languages. ICEIS: International Conference on Enterprise Information Systems, Apr 2014, Lisbon, Portugal. pp.298-306, 10.5220/0004895202980306 . lirmm-01276482

\section{HAL Id: lirmm-01276482 \\ https://hal-lirmm.ccsd.cnrs.fr/lirmm-01276482}

Submitted on 4 Feb 2020

HAL is a multi-disciplinary open access archive for the deposit and dissemination of scientific research documents, whether they are published or not. The documents may come from teaching and research institutions in France or abroad, or from public or private research centers.
L'archive ouverte pluridisciplinaire HAL, est destinée au dépôt et à la diffusion de documents scientifiques de niveau recherche, publiés ou non, émanant des établissements d'enseignement et de recherche français ou étrangers, des laboratoires publics ou privés.

\section{(이) $\$$}

Distributed under a Creative Commons Attribution - NonCommercial - NoDerivatives 44.0 


\title{
A Framework for Concurrent Design of Metamodels and Diagrams Towards an Agile Method for the Synthesis of Domain Specific Graphical Modeling Languages
}

\author{
François Pfister ${ }^{1}$, Marianne Huchard ${ }^{2}$ and Clémentine Nebut ${ }^{2}$ \\ ${ }^{1}$ LGI2P, Ecole des Mines d'Alès, site de Nîmes, Parc Scientifique G. Besse, 30000 Nîmes, France \\ ${ }^{2}$ LIRMM, CNRS, Université Montpellier 2, 161 rue Ada, 34095 Montpellier Cedex 5, France
}

\begin{abstract}
Keywords: Model Driven Architectures and Engineering, Modeling Formalisms and Notations, Domain Specific Languages, Graphical Syntax, Concrete Syntax.

Abstract: $\quad$ DSML (Domain Specific Modeling Languages) are an alternative to general purpose modeling languages (e.g. UML or SysML) for describing models with concepts and relations specific to a domain. DSML design is often based on Ecore metamodels, which follow the class-relation paradigm and also require defining a concrete syntax which can be either graphical or textual. In this paper, we focus on graphical concrete syntax, and we introduce an approach and a tool (Diagraph) to assist the design of a graphical DSML. The main principles are: non-intrusive annotations of the metamodel to identify nodes, edges, nesting structures and other graphical information; immediate validation of metamodels by immediate generation of an EMF-GMF instance editor supporting multi-diagramming. We report a comparison experience between Diagraph and Obeo Designer (a commercial proprietary tool), which was conducted as part of a Model Driven Engineering Course.
\end{abstract}

\section{INTRODUCTION}

Practitioners who model technical or sociotechnical systems master usual notations such as those proposed by UML (Omg, 2006). This language provides a finite number of concepts and notations for expressing structural and behavioral views of the systems under study. In some cases, the UML notations are too generic, and practitioners have to define ex nihilo a language which is able to handle their specific concepts. In this case, they often start with a classrelation formalism, either extending UML, or using MOF (Omg, 2006), a class based language, to define the concepts which were previously unavailable, so as to obtain a new language tailored to their field. Defining such a language thus includes two major phases: first, the abstract syntax is defined with the use of a class diagram, and second, a concrete syntax is proposed, to specify the form of (textual or graphical) statements that conform to the abstract syntax. In this paper we are interested in graphical concrete syntaxes. These have been somewhat neglected by language theorists: there is no consensus, as this exists with MOF for abstract syntaxes or EBNF (Garshol, 2003) for textual concrete syntaxes, about a descrip- tion language for graphical concrete syntaxes. Indeed, designing and implementing a graphical notation is a complex activity requiring significant expertise, both in its semiotic and cognitive concerns, and in its technical and operational aspects.

Several solutions for developing graphical syntaxes exist, but none of them satisfies all our needs. In particular, we are interested in a framework that offers at the same time: an easy to use solution, a native support of the multi-view paradigm, a native or easy support of nested nodes, an integration in the EclipseOSGI ecosystem, which is a de facto standard in the Model Based Engineering field, an open technology, with a published metamodel, MOF compliant, and a released tool (regularly updated).

In this paper, we propose a method and a tool (Diagraph) for agile development of graphical modeling languages on top of Ecore (Budinsky et al., 2003). The objective of Diagraph is to design Domain Specific Modeling Languages (DSML). These languages are closely tailored to elicit models representing complex systems in general, even beyond software engineering. Such models, even if they belong to a specific domain, must describe in general, structural concepts (composite things), operational concepts (work-

Pfister F., Huchard M. and Nebut C.

298 A Framework for Concurrent Design of Metamodels and Diagrams - Towards an Agile Method for the Synthesis of Domain Specific Graphical Modeling Languages.

DOI: $10.5220 / 0004895202980306$

In Proceedings of the 16th International Conference on Enterprise Information Systems (ICEIS-2014), pages 298-306

ISBN: 978-989-758-028-4

Copyright (C) 2014 SCITEPRESS (Science and Technology Publications, Lda.) 
flows, processes) that convert composite things or behaviors that define the states of the systems. The Diagraph framework provides a set of tools and processes that supports the design of these languages, by defining simultaneously their abstract syntax (metamodel) and their concrete syntax (notation and diagrams). The Diagraph description language allows us defining a new graphical modeling language by annotating the metamodel (abstract syntax). Diagraph includes the multiple view paradigm.

We also aim to integrate Diagraph into the Eclipse ecosystem, and to comply with the current standards of Model Based Engineering (MBE). Thus, we designed Diagraph as a technical overlay over GMF (Gronback, 2009), which is powerful but overly complex for the end user. In addition, we propose a process, which lacks in most of the existing propositions. In our context of academic research, we wish to propose an open tool and its related concepts at the disposal of the MBE community. At the end of this paper we relate an experiment, where we compare Diagraph and Obeo Designer (Juliot and Benois, 2009), a proprietary framework.

In Section 2, we study the existing state of the art. In Section 3 and 4 we present our contribution: A process in two phases, some key features of our tool, the Diagraph language, and its underlying metamodel. In Section 5 we give a use-case that typifies the problem of designing a graphical concrete syntax while designing the metamodel. In Section 6, we relate the experiment we did to compare Diagraph and Obeo Designer. We conclude in Section 7 and discuss future research directions.

\section{STATE OF THE ART}

In this section, we study frameworks which are able to generate graphic editors from which we can create models, that are instances of a given metamodel. Main inputs of the tools that generate such editors are the given metamodel and parameters provided by the modeling expert. The degree of automation of the generation process remains a challenge (Baetens, 2011). We list below some existing solutions.

GMF (Gronback, 2009) is a framework based on a mapping between Ecore, and Gef, a Graph Drawing engine which is a part of Eclipse (Eclipse, 2001). This framework is powerful, but poorly documented, and therefore requires a huge technical expertise, this results in a steep learning curve.

ModX (Renaux et al., 2005) is a graphic tool used to create both models and metamodels based on Ecore. It supports XMI format (import/export) in order to be interoperable with other MDA tools. The framework integrates the following functionalities: edition of metamodels, graphical notations (view types), and instance models. Several view types can be defined for a metamodel, and view types allow nested structures. ModX has an outstanding main feature: it is possible to change the underlying metamodel or one of its graphical notation while editing a related model. Even if ModX is Ecore compliant, the framework is a standalone tool, and thus not able to be integrated in contemporary tool chains based on plugin architectures such as the Eclipse (Eclipse, 2001) ecosystem.

MetaEdit+ (Kelly and Tolvanen, 2008) is not based on the Emf-Ecore stack, but on a specific metametamodel named GOPRR (Graph, Object, Property, Role and Relationship).

GME (Ledeczi et al., 2001) whose class graphical notation is, in addition, atypical is based on MS Component Object Model technology. Microsoft Dsl Tools has a proprietary meta-metamodel, while XMF Mosaic's is based on an infrastructure named XCore.

Obeo Designer (Juliot and Benois, 2009) is a modeling environment based on the notion of points of view as promoted by the IEEE1471 standard (IEEE, 2000). It can be integrated as a component of the Eclipse platform (Eclipse, 2001). The Obeo technology is based on EMF and GMF-Runtime. In addition to the diagrammatic notation, Obeo also represents models with tables and tree views. Obeo process begins by defining the vocabulary of the domain (concepts and their relationships, through a metamodel). During the second phase, the concrete syntax is built by creating diagram elements by means of a form based editor, and by associating these graphical elements with the metamodel elements. Styles are also associated with the graphical elements. Constraints on the model can be expressed in an OCL-like language. The user experience of Obeo Designer goes through capturing the concrete syntax by the mean of property editors, through a process, which is described in a user guide. That process is as complex as it needs a primary help given by Obeo's experts team. The underlying metamodel of Obeo Designer is not published nor documented. Obeo Designer and MetaEdit+ are commercial tools that are split in two different parts: a workbench, a tool for designing modeling languages and a modeler, a tool for using modeling languages.

While Diagraph is tightly integrated in the Eclipse platform, its principle is inspired by Eugenia (Kolovos et al., 2010), which consists to annotate the metamodel with concrete syntax statements, by the mean of EAnnotation objects. However, Eugenia is a 
Table 1: Features of available solutions.

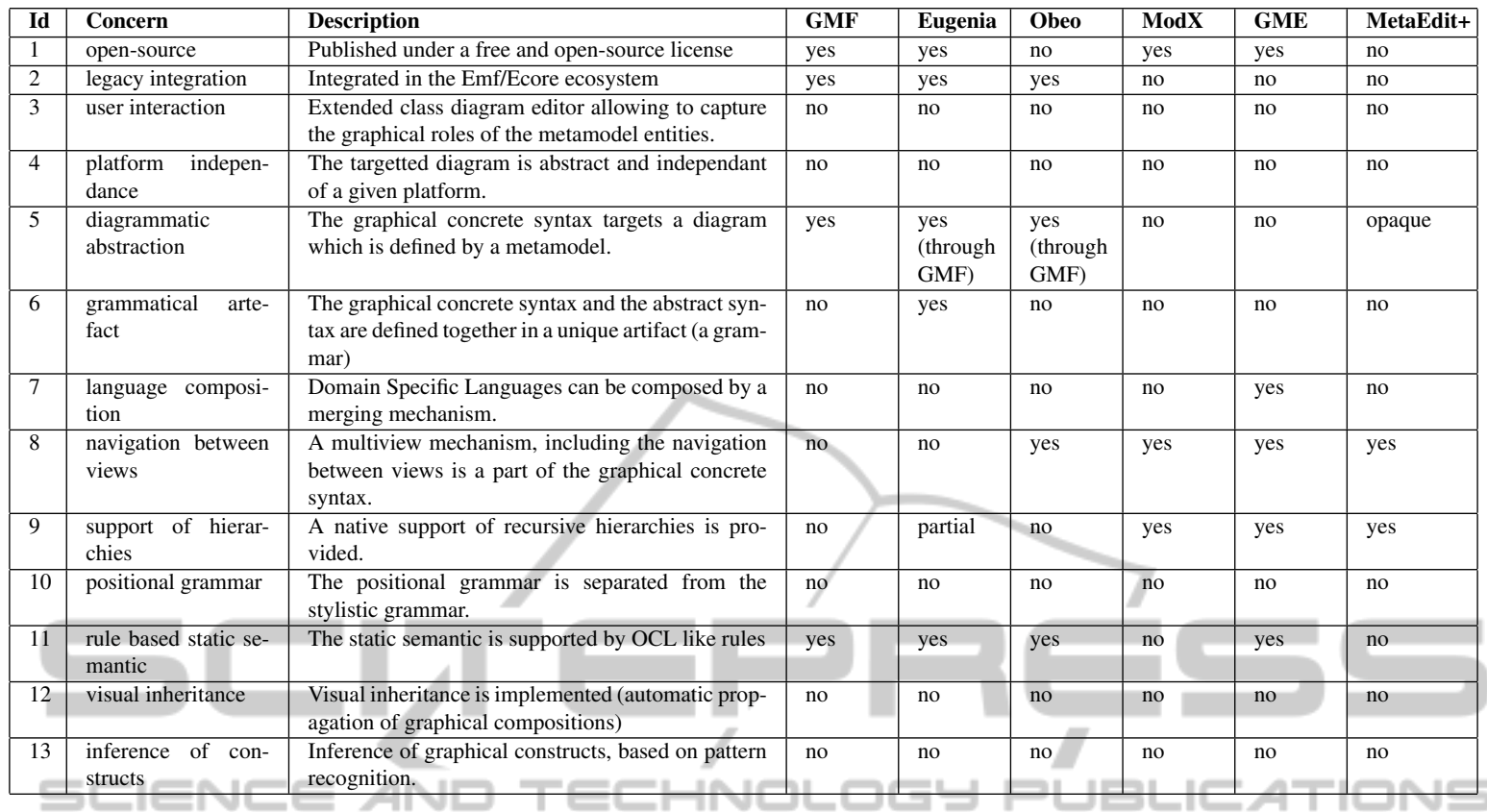

very thin layer over GMF, which hides the complexity of the latter, but, it does not provide large scale metamodel handling as does Obeo Designer.

As it results from the above survey, several limitations of the existing work lead us to design a new language and framework. The Table 1 summarizes some features available in the candidate frameworks. None of the latter reaches completely our requirements: an ideal tool would combine the ability to work simultaneously on the abstract syntax and the concrete syntax as does Eugenia (but not Obeo Designer), and manage multiple views as does the latter. As we will explain in the remainder of the paper, these 13 features are offered by Diagraph, our tooled method for designing Domain Specific Graphical Modeling Languages.

\section{METHODOLOGY}

In this section, we describe first the general process that we propose to DSML designers, and then the relations of Diagraph with several diagram renderers and graph drawing platforms.

\subsection{The Process}

In practice, designers are focusing on the DSML metamodel that defines the core concepts of the language. This phase is generally well controlled by practitioners, but is often separated from the design of graphical concrete syntax. DSMLs are bet- ter built in an incremental way, so an iterative approach is adopted, that allows many stake-holders and end-users to validate and verify the welformness of the language under study. Such an iterative and incremental process would be possible only with an adapted tool support. A defined workflow shows how to process temporally in parallel and with the same interest, the two aspects of the modeling process. Indeed, an impossibility or difficulty of graphically elicitating a concept coming from the metamodel may denote the transgression of a well-formedness rule, and even a conceptual error.

The process we propose organizes the design process of the DSML by maintaining consistency in its abstract syntax and concrete syntax graphs. The relationship between the abstract and concrete syntax graphs is a third graph of correspondences between the elements of both graphs. As we have seen, this correspondence graph is noted on the abstract syntax graph by annotating the latter with a very simple language, like a layer that would provide additional information to a map, by transparency. In addition, the process prescribes to compose a language with fragments located in a repository based on the concept of mega-model (Favre, 2006): each metamodel is stored with several sample models that are its instances, these models figure typical use cases, and that also are witnesses of their ability to be instanciated, and are a kind of factual proofs of their wellformedness. 


\subsection{The Framework}

The modeling framework, named Diagraph, supports the described process, the annotation language, and includes the mega-model manager. Diagraph contributes to the Eclipse platform (Budinsky et al., 2003) as a plugin, in a non-intrusive way, on the top of legacy tools.

The Diagraph Framework is a component conforming to the OSGI (Castro Alves, 2011) industrywide standard, plugged within the Eclipse Platform, contributing to already existing services (e.g. it uses the layout service of the Ecore Tools, through the OSGI protocol). The principle of the user interface is not that of a wizard, but of a grammar that defines the graphical concrete syntax upon the abstract syntax. The user is guided by the reference manual that describes the Diagraph language syntax, at one hand, and the messages (and in the future a code completion feature) generated by the Diagraph internal parser in case of syntax errors, while writing the Diagraph annotations in a compartment beneath the EClass compartments in the class diagram editor. As we explained, the idea is to define the concrete syntax declaratively by the mean of a grammatical notation.

\subsection{A Two-phases Process}

The first phase of the design process of a graphical notation is the positional phase: which are the nested nodes (included in a compartment)? What is the nesting depth? Which are the nodes associated with a graphical link? Which are the labels used for the elements of the notation? How many views will compose the language? Which semantic classes will compose each view? Which is the navigation path between the different views? The positional phase is critical in sense of the design art, and also in an architectural sense: Good architectures that simplify usage are complex to design. The second phase of the process, which has significant cognitive consequences, but that is technically more trivial, is the stylistic phase; it corresponds to the choice of symbols associated with elements of the representation: icon, shape, color. The chosen symbols have to denote the semantics of the model. The method we propose clearly separates these two phases. This separation is underpinned by the metamodel structure of Diagraph. Diagraph's semiotic language is based on the paradigm of cascaded styles.

\subsection{Key Feature}

The key feature of Diagraph is the management of the graphical complexity. The paradigm of composition by graphically nesting model elements (when they are semantically and structurally nested), is a key factor in the quality level of cognitive effectiveness. Many well known notations use actively such constructs: statecharts (Harel, 1987), package representation in UML, hierarchical functional models (Ross, 1977).

Defining a compartment rather than an external related node is done by changing one keyword of the Diagraph description language (ref becomes kref). The graph structure of the concrete syntax (considered as an abstract graph) is preserved when switching from nested nodes to associated nodes: further tooling that would handle pattern recognitions or similarity scoring between different models deals with the same computable graph structure (Falleri et al., 2008).

When the nesting becomes too deep, the positive effect of the nesting paradigm declines and disappears. It is then necessary to hide the nested details included and adopt the paradigm of additional view equivalent to a zoom on the hidden details. The view definition is a native part of the Diagraph Language. As with compartments, the abstract graph structure of the concrete notation is preserved.

The separation of the model into different views represents a System Architecture, conforming to the IEEE 1471 information model (Muller, 2009)).

\section{IMPLEMENTATION}

As does Eugenia (Kolovos et al., 2010), Diagraph annotates the metamodel with concrete syntax statements, by the mean of EAnnotation objects. However Diagraph is an entirely original development. Eugenia has some conceptual and usability limitations that have been addressed by Diagraph, where we have: visible key-value pairs acting as a visual stereotype mechanism, user interface integrated in the Ecore Tools editor, multi-view concept, separation of positional definition and stylistic definition.

The core difference is that Diagraph is based on a proper metamodel which describes the domain of diagramming, while Eugenia uses the metamodel of GMF. Therefore Diagraph is platform independent, and able to address other diagramming platforms (e.g. Graphviz).

\subsection{The Diagraph Metamodel}

The abstract syntax of the Diagraph language is a straightforward metamodel which conforms to a specialized graph. The Figure 1 shows a simplified version of the positional part of the Diagraph metamodel 


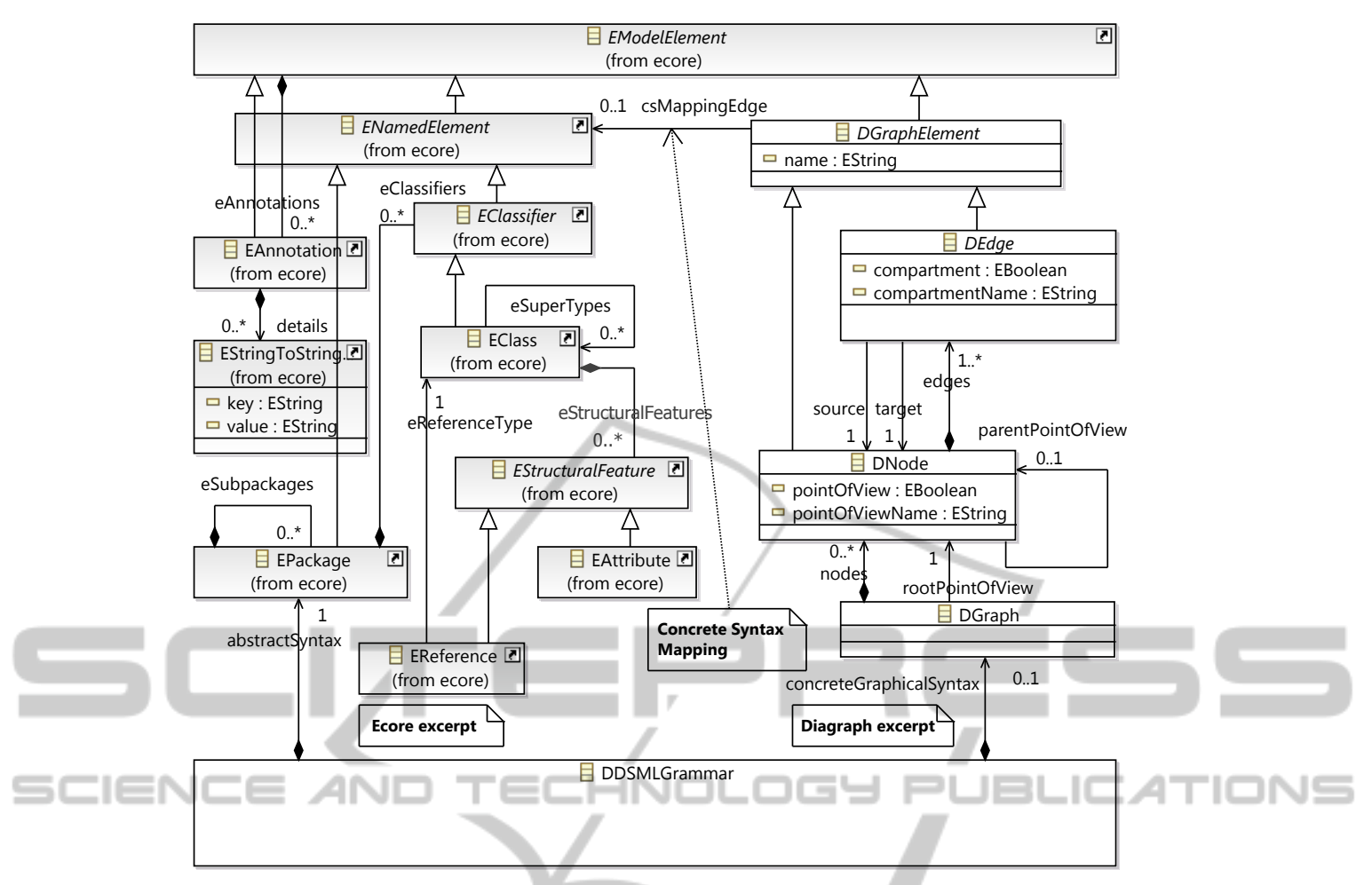

Figure 1: The positional part of Diagraph, related to Ecore (simplified excerpts).

(at the right) associated to the Ecore metamodel (at the left). The stylistic part is based on another independent metamodel that is here out of the scope, for the sake of clarity. The metamodel of Diagraph represents an abstract diagram which has a graph structure. Nodes can be graphically integrated into each other (if the attribute compartment is true). Elements of the concrete syntax (DGraphElement) belonging to the diagram are mapped by the csMappingEdge reference to elements belonging to the Ecore metamodel (ENamedElement). That principle of mapping a concrete syntax onto an abstract syntax is also described by (Brambilla et al., 2012) in their section 7.4.1, and our metamodel is close to a pattern called "generic gcs metamodel" by these authors. The latter distinguishes Mapping-centric Graphical Concrete Syntaxes from Annotation-centric Graphical Concrete Syntaxes. However, our approach starts from an annotation-centric syntax, to generate an instance of the Diagraph metamodel, mapped onto the metamodel of the target domain. That Diagraph instance is a graphical notation, that is to say a concrete syntax, which is at this stage independent of a graphical renderer. The renderer can be, on one hand, the GMF Runtime, invoked by a diagram editor resulting from a transformation taking a Diagraph instance as an input and producing the GMF tools artefacts, or on the other hand, the Graphviz Runtime, activated by a DOT model resulting from a transformation taking a Diagraph instance as an input. Our proposition is annotation-centric in the sense of (Brambilla et al., 2012), and also wraps an internal mapping-centric approach.

The diagram itself will be generated by a DGraph instance, while nodes (either top nodes, or child nodes nested in other nodes) will result of DNode instances. A DNode may be the root of a point of view, so a new diagram is opened starting from this DNode if the attribute pointOfView is true. A DNode is always underpinned by an EClass, in this case (csMappingEdge relates to an EClass).

A DEdge object will give an edge on the diagram. Three different kinds of edges may be created:

- The DEdge is underpinned by an EClass, thus it carries labels taken from the EClass attributes, when the csMappingEdge relates to an ENamedElement which is an EClass.

- The DEdge is underpinned by an EReference, when the csMappingEdge relates to an ENamedElement which is an EReference. This leads to two cases:

- The DEdge is a nesting edge (the compartment attribute is true), so the target DNode is graphically nested within the source DNode (and the 
Table 2: The Diagraph vocabulary.

\begin{tabular}{|c|c|c|}
\hline Keyword & Semantics & $\begin{array}{l}\text { Abstract Syntax correspon- } \\
\text { dence }\end{array}$ \\
\hline node & $\begin{array}{l}\text { A graphical shape plays } \\
\text { the role of the related class }\end{array}$ & DNode \\
\hline link & $\begin{array}{l}\text { A graphical line plays the } \\
\text { role of the related class } \\
\text { (noted on the class) }\end{array}$ & DEdge \\
\hline $\operatorname{lnk}$ & $\begin{array}{l}\text { Idem, but acts as a short- } \\
\text { cut noted on the source } \\
\text { class }\end{array}$ & DEdge \\
\hline lsrc & $\begin{array}{l}\text { Defines the source for a } \\
\text { link }\end{array}$ & DEdge.source \\
\hline ltrg & Defines a target for a link & DEdge.target \\
\hline cont & $\begin{array}{l}\text { Defines a container for a } \\
\text { link }\end{array}$ & DNode.edges \\
\hline cref & $\begin{array}{l}\text { A graphical nested com- } \\
\text { partment holds the target } \\
\text { node }\end{array}$ & DEdge.source (opp \\
\hline kref & $\begin{array}{l}\text { Idem, but several compart- } \\
\text { ments are created, one per } \\
\text { type }\end{array}$ & DEdge.source (opposite of) \\
\hline afx & $\begin{array}{l}\text { A graphical affixed port is } \\
\text { the target node }\end{array}$ & DEdge.source (opposite of) \\
\hline$\Longrightarrow$ & $\begin{array}{l}\text { A graphical line plays the } \\
\text { role of the related refer- } \\
\text { ence (no underlying class, } \\
\text { thus no label can decorate } \\
\text { the line) }\end{array}$ & DEdge.source (opposite of) \\
\hline pov & $\begin{array}{l}\text { A new diagram plays the } \\
\text { role of the node }\end{array}$ & DNode.pointOfView \\
\hline view & Identifies the diagram & DNode.pointOfViewName \\
\hline nav & $\begin{array}{l}\text { A graphical hyperlink } \\
\text { leads to a new Diagram } \\
\text { opened as a view }\end{array}$ & DEdge.target \\
\hline label & $\begin{array}{l}\text { A graphical label deco- } \\
\text { rates the node or the link, } \\
\text { the label plays the role of } \\
\text { the related attribute refer- } \\
\text { enced by the argument }\end{array}$ & DGraphElement.labelAttribute \\
\hline
\end{tabular}

DEdge is graphically hidden).

- The DEdge is a simple relation, so the target DNode will be shown as a sibling node on the diagram, and a visible line generated by the DEdge will be shown, without carrying any label (there is no EClass providing any attribute).

\subsection{Stylistic Part}

The stylistic part has a tree structure and can be instantiated as a cascadable styles model. It is not shown in this paper (the stylistic concern is more trivial than the positional one, even if our proposition of a cascadable mechanism similar to the principle of cascading style sheets seems, as far we know, original when applied to the field of diagramming).

\section{CASE STUDY}

To illustrate the paper, we propose a toy case which is that of a publication process whose concepts are captured in a metamodel shown in Figure 2 and 3 . An instance model is shown in Figure 5.

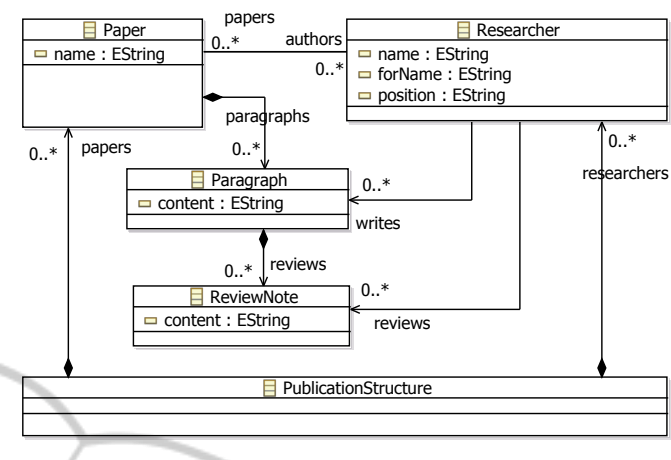

Figure 2: Abstract Syntax of the Publication Domain (structural part).

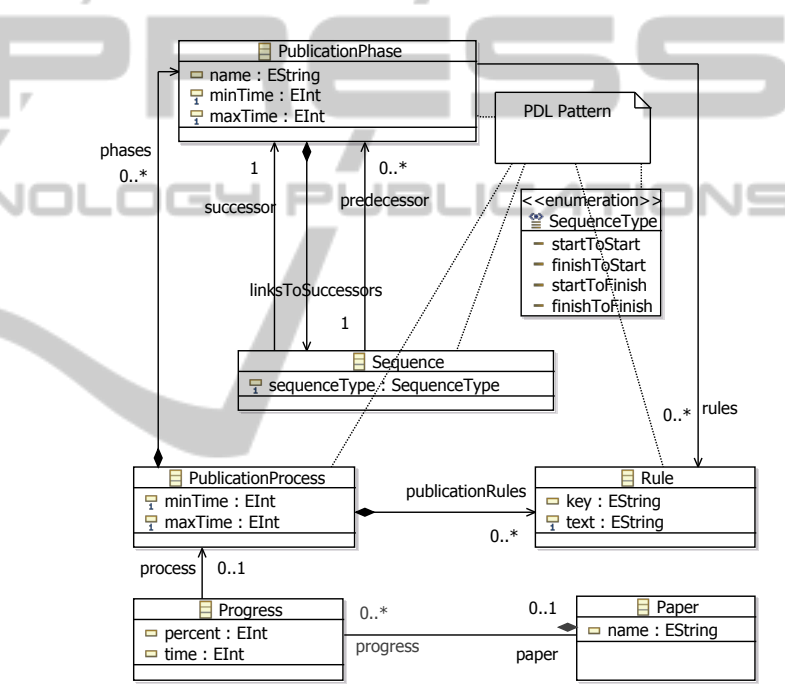

Figure 3: Abstract Syntax of the Publication Domain (process part).

\subsection{A Language for Publications}

The elements of the domain are as follows: PublicationProcess initiated by a research team, PublicationStructure composed by several Researcher, shown Figure 2. The metamodel is split in two parts corresponding, in the resulting concrete syntax, to two different views, a static (structural) view, and a dynamic (process) view. The PublicationStructure holds many papers (Paper), each composed of paragraphs (Paragraph), and each paragraph of reviews (ReviewNote). One Researcher writes many papers and one Paper has several authors. One Researcher writes many paragraphs and reviews them with ReviewNote. The PublicationProcess (Figure 3 ) is the root of the process view, that part of the metamodel implements the PDL pattern 


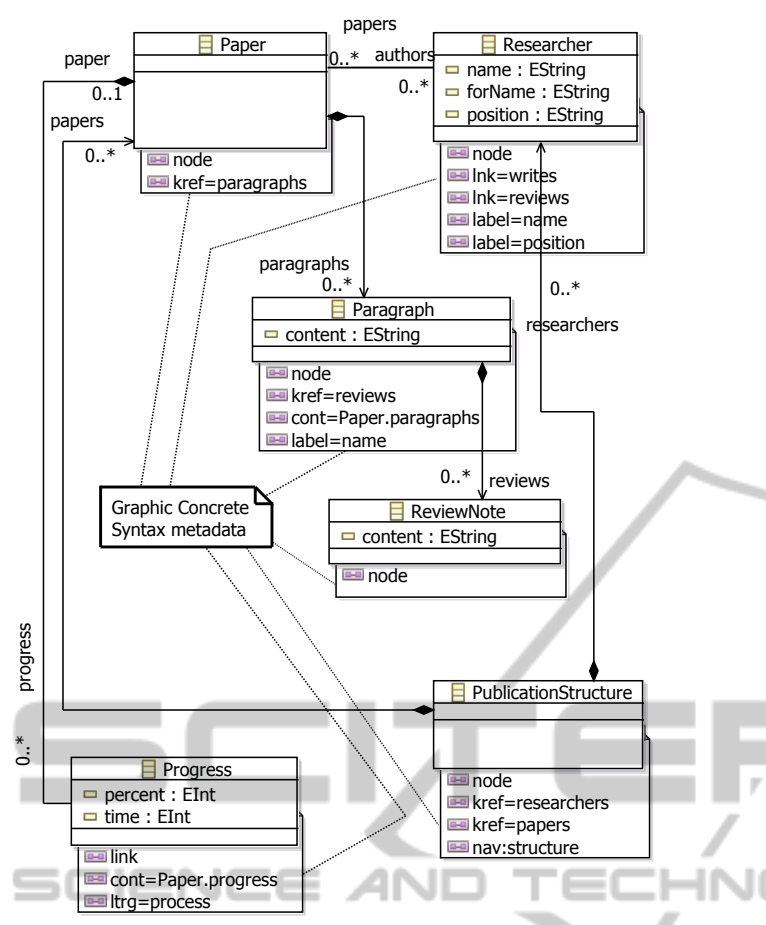

Figure 4: The concrete syntax annotated onto the abstract syntax (fragment).

proposed by (Combemale et al., 2007). The two parts of the metamodel are related by two EReferences: neededPerson, and process. So to obtain the best expressiveness for our graphical language, we need to design it according to the criteria proposed by Moody. (Moody, 2009). These criteria consider, first, the relative position of the graphic symbols that make up the graphical language, and then, stylistic criteria about shapes, icons, colors and line styles. We are interested, in this case, only on the positional criterion. One possible notation is that shown in Figure 5.

To specify graphical positional roles for the elements composing a metamodel, we define in Table 2 the Diagraph vocabulary, and the associated semantics. The third column, Abstract Syntax correspondence, refers to the Diagraph metamodel shown Figure 1.

We use this language to annotate our metamodel as shown in Figure 4. For example, Paper will be represented as a node, while Progress will be represented by a link. The annotation area uses the EAnnotation from Ecore. The EAnnotation cartridge is automatically laid out exactly beneath the EClass rectangles, figuring an extension of the class diagram (Diagraph contributes to the layout service within the Eclipse platform by providing its specific layout constraints). The vocabulary of the concrete syntax definition is directly derived from the metamodel in Figure 1 as shown in the third column of Table 2.

The notation, when embodied in a model instance of the Publication metamodel augmented by the Diagraph model named publication.diagraph, looks like Figure 5. At this point, the style has not yet been applied. The positional criterion is a key point that guarantees the cognitive fit of a notation, and the semiotic (stylistic) concern will further improve the result. The stylistic part is not shown in this paper, for the sake of clarity, but it follows the same principle of an annotation based language.

\section{EXPERIMENT}

We evaluated the usability of Diagraph through a Master modeling course given at the University of Montpellier II. We asked students to implement several simple graphical languages (see metamodels at http://aigle-2012.googlecode.com/svn/experiment) with each tool. We evaluated the performance of each tool. This process was based on an agility scheme. After an introduction to the overall implementation of the Eclipse EMF and GMF Tooling, two workshops were proposed to build Graphical Domain Specific Modeling Languages based on simple metamodels, each consisting of 6 classes, representing a toy modeling situation, with at least one compartmentalized node, several un-typed relations without any label, and also several typed links carrying labels. The multi-view feature was not processed due to the limited time available. The metamodels are different for each tool compared, and for each mode (supervised and autonomous). However, they are of comparable complexity in terms of the metamodel structure. The profile of the subjects is a mix of research profile and professional profile. For each experiment, each student restitutes:

- A free report outlining the taken steps, including text and screenshots of the experiment in progress.

- An assessment questionnaire for the process.

At the end of the experiment, an interview was conducted. The students consider that the fact of being based on a annotation language is finally more a disadvantage for Diagraph. They think that using annotations, which are informal metadata, are not adapted to write a formal language. The unavailability of a wizard, a property editor, a code completion mechanism also was a disadvantage for Diagraph. On the other hand, they consider that the cognitive comprehension is in favor of Diagraph which is intuitive, due to the proximity of the abstract syntax which is close to the graphical concrete syntax, in a unique artifact. 


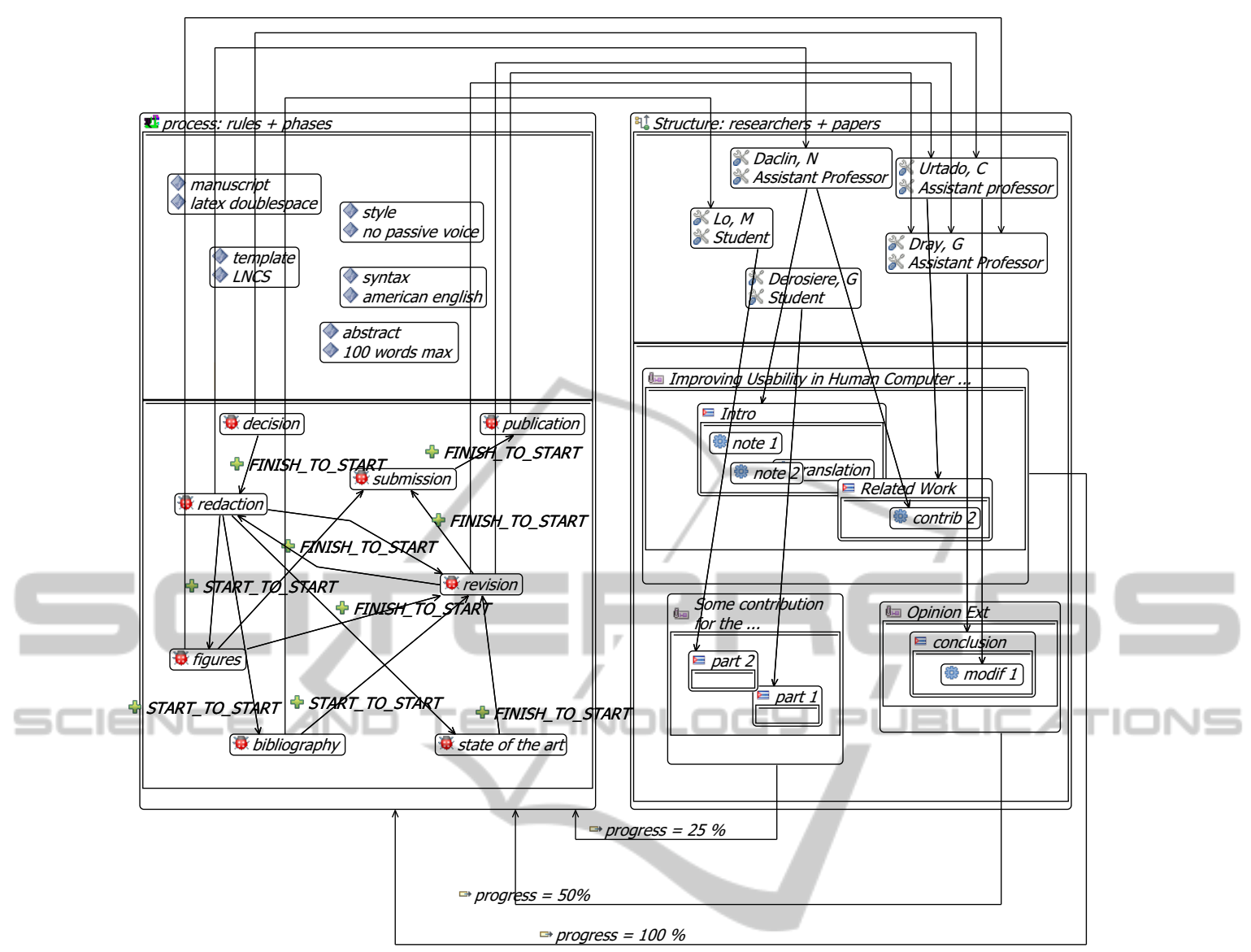

Figure 5: A Publication model created with the generated diagram editor.

Similarly, they believe that the syntax of Diagraph is very flexible, e.g. it is easy to convert an independent node into a nested node, by changing a keyword, while they must undo a complex construct to do the same operation with Obeo. Despite the difficulty of editing Diagraph expressions by the mean of non syntactically controlled annotations, they feel that the design process is faster with Diagraph.

In the case of Obeo Designer, they are close to the concrete syntax, but far from the abstract syntax which resides in a different instance of Eclipse. In the participant's mind, Diagraph is, for the moment, an experimental tool, close to the research area, while Obeo is adapted to industrial contexts. This fact is coherent with the nature of the license, which is expensive in the case of Obeo while Diagraph will have a free open-source license. We notice that Obeo procures academic free licenses; however, the users complain that Obeo is a black box.

\section{CONCLUSION}

We have proposed Diagraph, a modeling framework that helps the design of graphical Domain Specific Modeling Languages - DSML, on the top of Eclipse EMF-GMF. It includes, on the one hand a process, and the other hand a set of annotations to enrich a simple Ecore conformant metamodel with concrete syntax graphical roles, playing in one single model the role of a grammar for graphical languages as EBNF does for textual languages. Taking this grammar as an input, Diagraph automatically generates all EMFGMF artifacts (intermediate models, diagram editors, skeletons of unit tests). Multi-diagramming is natively supported. Diagraph is non-intrusive: no dependency is left in the target models; the framework is structured in a platform independent layer based on a clean metamodel, defining an abstraction for graphical concrete syntax, and a transformation layer targeting the GMF platform, but also other graphdrawing or diagramming systems: Dot-Graphviz (yet implemented), Graphiti, GraphML (non limitative 
list, and not yet implemented). The tool includes a "zoo" (Favre, 2006) of numerous example cases. The Diagraph is released under an open-source license at http://code.google.com/p/diagraph/.

Our current work is to design functional models such as eFFBD (Pfister et al., 2012), which are hierarchical decompositions in the sense of IDEF0 (Ross, 1977), and also are executable models (timed Petri nets), even if the operational semantics is not a part of our research.

Abstract syntax and concrete syntax coevolution is realized de facto by Diagraph. As a future work, we would like to master the coevolution of a language (abstract and concrete syntax) and existing instances of that language.

We also would like to (semi) automatize the generation of a Diagraph grammar, by analyzing patterns in the abstract syntax. Such patterns would drive the concrete syntax: in fact they are concrete-syntax intentions. We think about integrating the annotation mechanism in the refactoring process of Eclipse, and to improve the editor with code completion. A longterm objective is building concrete syntaxes by abstract syntax patterns recognition, but also, at a different (lower) meta-level, building model by imitating model patterns taken from a thematic repository. Another objective is to improve the megamodel management by splitting the abstract syntax in several metamodels. At last, we would like promoting the Diagraph metamodel at the M3 level.

\section{REFERENCES}

Baetens, N. (2011). Comparing graphical DSL editors: AToM3 , GMF, MetaEdit+. Technical report, University of Antwerp, Antwerp, Belgium.

Brambilla, M., Cabot, J., and Wimmer, M. (2012). ModelDriven Software Engineering in Practice. Morgan Claypool.

Budinsky, F., Steinberg, D., Merks, E., Ellersick, R., and Grose, T. J. (2003). Eclipse Modeling Framework (The Eclipse Series).

Castro Alves, A. (2011). OSGi in Depth. Manning.

Combemale, B., Garoche, P.-L., Crégut, X., and Thirioux, X. (2007). Towards a Formal Verification of Process Model's Properties - SimplePDL and TOCL case study. In 9th International Conference on Enterprise Information Systems ICEIS, pages 80-89. INSTICC press.

Eclipse (2001). About the Eclipse Foundation.

Falleri, J.-R., Huchard, M., Lafourcade, M., and Nebut, C. (2008). Meta-model Matching for Automatic Model Transformation Generation. In MODELS'08: 11th International Conference on Model Driven Engineering Languages and Systems, volume 5301, pages 326340.
Favre, J.-M. (2006). Megamodelling and Etymology. In Transformation Techniques in Software Engineering. Schloss Dagstuhl - Leibniz-Zentrum für Informatik.

Garshol, L. M. (2003). BNF and EBNF: What are they and how do they work?

Gronback, R. (2009). Eclipse Modeling Project: A DomainSpecific Language (DSL) Toolkit. Addison-Wesley Professional.

Harel, D. (1987). Statecharts: A visual formalism for complex systems. Sci. Comput. Program., 8(3):231274.

IEEE (2000). IEEE SA - 1471-2000 - IEEE Recommended Practice for Architectural Description for SoftwareIntensive Systems.

Juliot, E. and Benois, J. (2009). Viewpoints creation using Obeo Designer or how to build Eclipse DSM without being an expert developer?

Kelly, S. and Tolvanen, J.-P. (2008). Domain-Specific Modeling. Wiley-IEEE Computer Society Press.

Kolovos, D., Rose, L., Abid, S., Paige, R., Polack, F., and Botterweck, G. (2010). Taming EMF and GMF Using Model Transformation. In Petriu, D., Rouquette, N., and Haugen, O., editors, Model Driven Engineering Languages and Systems, chapter 15, pages 211225. Springer, Berlin / Heidelberg, lecture no edition.

Ledeczi, A., Maroti, M., Bakay, A., Karsai, G., Garrett, J., Thomason, C., Nordstrom, G., Sprinkle, J., and Volgyesi, P. (2001). The generic modeling environment. In Workshop on Intelligent Signal Processing.

Moody, D. (2009). The "Physics" of Notations: Toward a Scientific Basis for Constructing Visual Notations in Software Engineering. IEEE Transactions on Software Engineering, 35(6):756-779.

Muller, G. (2009). How to create an architecture overview.

Omg (2006). Meta Object Facility (MOF) Core Specification. Technical report.

Pfister, F., Chapurlat, V., Huchard, M., Nebut, C., and Wippler, J.-L. (2012). A proposed meta-model for formalizing systems engineering knowledge, based on functional architectural patterns. Systems Engineering.

Renaux, E., Le Pallec, X., and Moura, C. (2005). ModX a graphical tool for MOF metamodels. In European Conference on Model Driven Architecture - Foundations and Applications.

Ross, D. T. (1977). Structured Analysis (SA): A Language for Communicating Ideas. IEEE Transactions on Software Engineering, 3(1):16-34. 